\title{
Heavy-ion physics at high baryon densities
}

\author{
Volker Friese ${ }^{1, a}$ \\ ${ }^{1}$ GSI Darmstadt
}

\begin{abstract}
Currently, several experimental programmes, both at existing and at future accelerator facilities, aim at investigating strongly interacting matter with nuclear collisions at energies below top SPS energy. These activities complement the heavy-ion experiments conducted at the highest available energies at the RHIC and LHC accelerators. In this report, we discuss the motivation for and prospects of the low-energy heavy-ion programmes.
\end{abstract}

\section{Studying QCD with nuclear collisions}

Collisions of heavy nuclei in the laboratory are the experimental tool to study nuclear matter off its ground state. In such collisions, a transient state of heated and compressed matter is produced, which allows to address two basic features of Quantum Chromo-Dynamics (QCD): confinement and chiral symmetry. In the low energy regime, where the coupling is strong, the constituents of QCD, quarks and gluons, are bound into hadrons. At momentum transfers larger than the intrinsic QCD energy scale $\Lambda_{Q C D} \approx 200 \mathrm{MeV}$, quarks and gluons are weakly bound, such that a transition from a hadronic medium to a Quark-Gluon Plasma (QGP) is expected once a certain energy density is reached. Similarly, it is expected that the approximate chiral symmetry of QCD, spontaneously broken in the ground state, is restored at high energy densities, leaving only the explicit symmetry breaking by the non-vanishing Higgs masses of the quarks. This qualitative considerations are substantiated by calculations solving QCD on a space-time lattice, which show a transition to deconfined and chirally symmetric matter at a critical temperature $T_{c} \approx 170 \mathrm{MeV}$ at vanishing net-baryon density [1].

Experimentally, the study of nuclear matter under extreme conditions by colliding heavy ions has a history of more than four decades by now. Since first experiments at the Bevalac in the early 1970s, progress in the field was achieved by increasing the collision energy through the availability of new, ever-enlarged accelerators: GSI-SIS, BNL-AGS, CERN-SPS, BNL-RHIC and, lately, CERN-LHC. It is widely accepted that a Quark-Gluon Plasma is created at the RHIC collider at a collision energy of $\sqrt{s_{\mathrm{NN}}}=200 \mathrm{GeV}$ [2]. With the advent of heavy-ion data from the LHC, is it possible to study the properties of the QGP in detail.

However, several experimental programmes, both currently running ones at existing accelerators as well as future projects at new experimental facilities, aim at investigating heavy-ion reactions at lower energies. The Beam-Energy Scan programme (BES) at RHIC, mainly conducted by the STAR experiment, studies nuclear collisions down to $\sqrt{s_{\mathrm{NN}}}=7.7 \mathrm{GeV}$ [3]. A first phase of this programme already took place in 2010 - 2014; a second phase is planned for 2018 - 2019. The NA61/SHINE

\footnotetext{
ae-mail: v.friese@gsi.de
} 
experiment at CERN-SPS, using an upgrade of the NA49 detector, addresses the energy range from $p_{\text {lab }}=13 \mathrm{~A} \mathrm{GeV}$ up to the top SPS energy $\left(p_{\text {lab }}=158 \mathrm{~A} \mathrm{GeV}\right)$, with focus on the collisions of lighter nuclei [4]. A similar energy range $\left(\sqrt{s_{\mathrm{NN}}}=4-11 \mathrm{GeV}\right)$ will be covered in collider mode by the planned MPD experiment at the new accelerator facility NICA at JINR Dubna, coming into operation from 2019 on [5]. The Compressed Baryonic Matter experiment (CBM) at the FAIR accelerator complex, currently under construction at GSI Darmstadt, will study nuclear collisions at even lower energies $\left(p_{\text {lab }}=2 A-45 A \mathrm{GeV}\right)$, with emphasis on very high interaction rates and, consequently, extremely rare probes [6]. The start of CBM data taking is expected for 2021. In addition, a new experiment NA60' at CERN-SPS is proposed, using the successful concept of NA60 to study dimuon production in heavy-ion reactions in the entire SPS energy range [7]. Under discussion is also a heavy-ion programme at the J-PARC facility in Japan [8]. To understand the motivation for these experimental activities, it is necessary to recall that a transition to deconfined and chirally symmetric matter can be reached not only by heating, but also by compressing matter.

\section{Exploring the QCD phase diagram}

In thermodynamic equilibrium, QCD matter can be characterized by two parameters, namely the temperature $T$ and the net-baryon density $\rho_{B}$ or its conjugate variable, the baryo-chemical potential $\mu_{B}$. Figure 1 shows a contemporary view of the phase diagram of strongly interacting matter in terms of these variables [9]. Cold nuclear matter - as found in normal nuclei with net baryon density equal to unity - consists of nucleons only. At moderate temperatures and densities, nucleons are excited to short-lived states (baryonic resonances) which decay by the emission of mesons. At higher temperatures also baryon-antibaryon pairs are created. This mixture of baryons, anti-baryons and mesons, all strongly interacting particles, is generally called hadronic matter, or baryonic matter if baryons prevail. At very high temperatures or densities the hadrons melt, and the constituents, the quarks and gluons, form new phases. More exotic phases, like colour superconductivity formed by correlated quark-quark pairs, are speculated about at low temperatures and very high net-baryon densities. Such matter may exist in the cores of neutron stars.

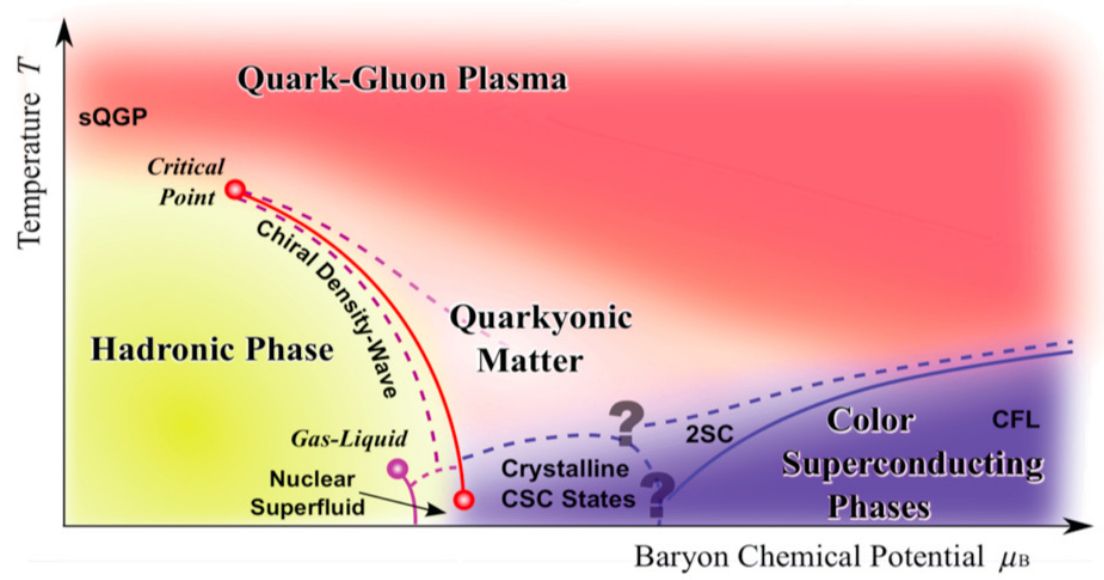

Figure 1: Sketch of the phase diagram of strongly interacting matter [9] 
Results of Lattice QCD indicate that at zero net-baryon density, close to the $T$ axis, the transition from hadronic to deonfined matter is of the cross-over type, meaning that the two phases are indistinguishable at $T_{c}$ [1]. Such calculations, however, are currently restricted to vanishing or small net-baryon densities. At higher densities, one has to rely on effective, QCD-inspired models. Such models predict the chiral transition to be of first order at higher $\mu_{B}$. Consequently, the regions of firstorder phase transition and of cross-over must be separated by a critical point, where the transition is a second-order one. Moreover, while at $\mu_{B}=0$, the chiral and deconfinement transitions seem to coincide, this need not be the case at higher baryo-chemical potentials. A non-congruence of both transition lines would give rise to a third phase, the so-called Quarkyonic matter, which is suggested by QCD models in the large $N_{C}$ limit [10].

The potentially rich structure of the QCD phase diagram at high net-baryon densities as suggested by theory surely calls for experimental verification. The discovery of the prominent landmarks like first- order chiral and deconfinement phase transitions and the critical point would be a major breakthrough in our understanding of the properties of nuclear matter.

Experimental results on hadron abundances produced in heavy-ion collisions indicate that the final state of such collisions is in or close to chemical equilibrium. Yields or yield ratios are successfully fitted by the statistical model, having temperature $T$ and baryo-chemical potential $\mu_{B}$ as free parameters[11]. The extracted values of these parameters, however, depend on the collision energy: they form a "freeze-out" line as shown in Fig. 2. Although it should be noted that these data points characterize only the final state of the collision, they indicate that different regions of the QCD phase diagram are accessible by varying the collision energy. While nuclear collisions at the highest available energies (RHIC and LHC) appear to probe matter at high temperatures and small $\mu_{B}$, the energy range below top SPS energy is suited for the study of the regime of high net-baryon density. The reason for this lies in the nature of the interaction process: at lower energies, the incoming barons are partially stopped in the interaction zone, creating a "fireball" with non-vanishing net-baryon content. In contrast, at high energies, the nuclei become transparent, such that the colliding nucleons leave the interaction zone after depositing a part of their kinetic energy. Consequently, a medium with vanishing net-baryon density is created at mid-rapidity.

The conjecture that matter with high net-baryon density is created in nuclear collisions at low SPS energies is supported by results of microscopic transport models, allowing to trace the evolution of the collision system. As shown in Fig. 3, different flavors of this model qualitatively agree that densities several times higher than saturation density are reached at such energies [12]. The maximum net-baryon density is expected for collisions at $30 \mathrm{AGeV}$ on a stationary target, corresponding to $\sqrt{s_{\mathrm{NN}}} \approx 8 \mathrm{GeV}$ (see Fig. 4).

The experimental questions addressing QCD matter at high net-baryon density are:

- What is the equation of state of matter at extreme baryon density? Does the statistical model still describe the hadron yields resulting from nuclear collisions at low energies, i.e., is the created matter still at chemical equilibrium? Recent results from the HADES experiment show puzzling deviations from a statistical description at very low energy $\left(E_{\text {beam }}=1.76 \mathrm{~A} \mathrm{GeV}\right)$ in the relatively light system $\mathrm{Ar}+\mathrm{KCl}[15]$.

- Where and of which type is the transition from hadronic to partonic matter at high $\mu_{B}$ ? Results from the SPS experiments suggest that a deconfined state is already created at top SPS energy [14]. If that holds true: at which energy is deconfinement first reached? Should the transition be of first order, there must be a co-existence phase with latent heat. The phase transition thus should manifest in discontinuities in the excitation functions of sensitive observables, such as yields, spectra and flow of strange or charmed hadrons and of lepton pairs. NA49 has reported such discontinuities as indications for an onset of deconfinement at around $30 \mathrm{~A} \mathrm{GeV} \mathrm{[16].} \mathrm{Although} \mathrm{this} \mathrm{data} \mathrm{interpretation}$ 


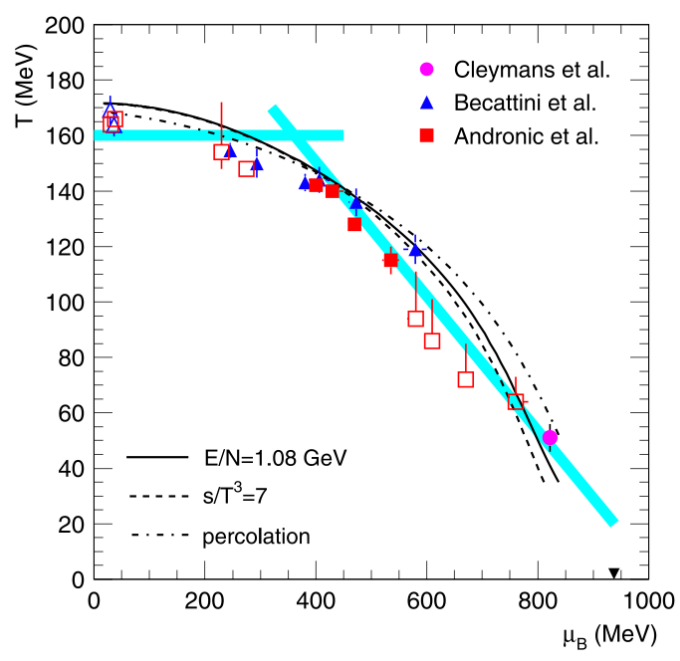

Figure 2: Freeze-out properties of the matter created in heavy-ion reactions at various collision energies [11]

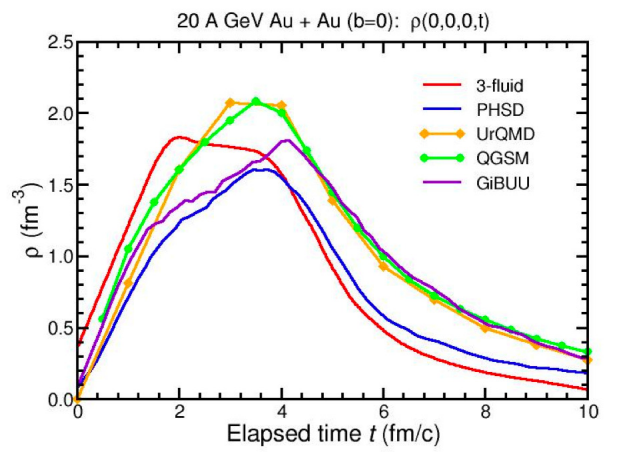

Figure 3: Baryon density in the centre of the reaction volume for $\mathrm{Au}+\mathrm{Au}$ collisions at $20 \mathrm{~A} \mathrm{GeV}$ as obtained from various microscopic transport models and from a 3-fluid hydrodynamic calculation [12]

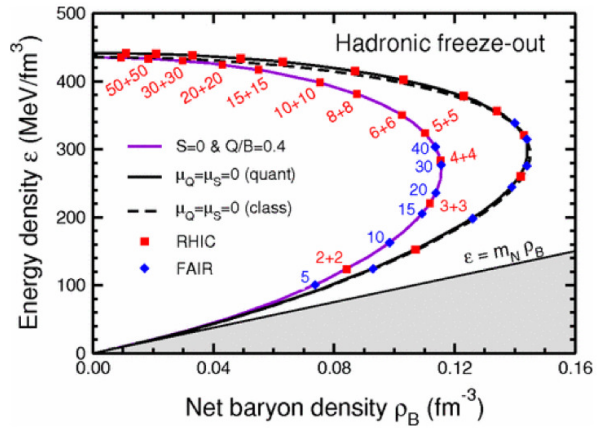

Figure 4: Freeze-out points of heavy-ion collisions in the energy density - net baryon density plane as resulting from a parametrization of the results of statistical model fits to measured hadron abundances [13]

is still under debate, it shows that the low SPS energy regime is a promising region for the study of the phase diagram.

- Is there a critical point and if yes, where? A critical point would be a consequence of a first-order phase transition at large $\mu_{B}$ and a cross-over at $\mu_{B}=0$. There is little guidance from theory as to its location in the phase diagram. It should manifest in large event-by-event fluctuation of conserved quantities such as net charge or baryon number. 
- Is there a Quarkyonic phase as suggested by Ref. [10]? In such a phase, chiral symmetry would be partially restored but confinement would still hold. It should be accessible be heavy-ion reactions in the low SPS energy regime. A first-order phase transition from hadronic to Quarkyonic matter should result in discontinuities of final-state observables like hadron yields and spectra, similarly to a phase transition to the QGP. Moreover, the restoration of chiral symmetry will reflect in the modification of hadron properties like mass and width. These can be studied e.g. by the decay of short-lived vector mesons.

These experimental topics call for systematic studies in terms of both collisions energy and size of the colliding system, which can be controlled by the centrality of the collision of heavy nuclei or by colliding lighter ion species. Up to date, only the bulk particles were measured in heavy-ion collisions below top SPS energy. Data from previous experiments at AGS and SPS on diagnostic probes of the dense stage of the fireball, such as multi-strange baryons, lepton pairs and charmed particles, are either scarce or lacking at all. These will be explored by the experimental programmes mentioned above, in particular with new-generation experiments at the upcoming accelerator facilities. In the following, we briefly discuss some of the observables suitable to provide information on the nature of the dense matter created in the fireball.

\section{Observables}

\subsection{Multi-strange hyperons}

The yields and phase-space distributions of multi-strange hyperons $(\Xi, \Omega)$ and are particularly promising tools to study the properties and the degrees of freedom of QCD matter at high densities and a possible transition to Quarkyonic matter. In nuclear collisions $\Xi^{-}$and $\Omega^{-}$can be created even below threshold (3.7 GeV for $\Xi^{-}, 7.0 \mathrm{GeV}$ for $\Omega^{-}$) via strangeness exchange reactions like $\Lambda \Lambda \rightarrow \Xi^{-} p$ and $\Lambda \Xi^{-} \rightarrow \Omega^{-}$, with the $\Lambda$ being previously produced in independent reactions. Alternatively, threebody collisions involving $\Lambda$ or kaons open new production channels for $\Xi$ and $\Omega$ with respect to $p+p$ reactions. The production of multi-strange hyperons is thus expected to be enhanced at high densities, and their yield to be sensitive to both the strangeness content and the baryon density reached in the fireball.

The production of anti-hyperons, on the other hand, is strongly suppressed in a baryon-dense environment. This constraint would be relaxed in case of a QGP, as demonstrated in Fig. 5, showing predictions of the $\Xi^{+}$yield at $160 \mathrm{~A} \mathrm{GeV}$ from the (hadronic) HSD transport model and the pHSD model, which includes a QGP phase. The difference is even more pronounced at lower energies: it amounts to a factor of eight for $\Omega^{+}$in central $\mathrm{Au}+\mathrm{Au}$ collisions at $25 \mathrm{~A} \mathrm{GeV}$.

Experiments addressing hyperon production must have good vertexing capabilities and high statistics. Data can be expected from the MPD experiment at NICA as well as from the fixed-target programme BM@N at the JINR Nuclotron. Because of the very low yield, systematic measurements of anti-hyperons production will be possible only with the CBM experiment with its high rate capability.

\subsection{Fluctuations and the critical point}

The observable to detect critical behaviour as expected in the vicinity of the QCD critical point are event-by-event fluctuations of conserved quantities. The first phase of the STAR beam energy scan programme was devoted to the search of such phenomena. No conclusive indications for criticality have yet been observed [3]. It should be noted that not only the location of the critical point is unknown, but it is also unclear how close to this point the system has to pass such that critical behaviour 


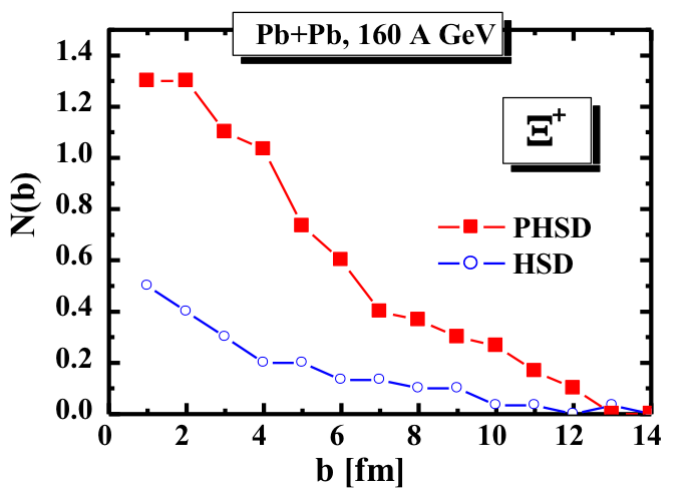

Figure 5: $\Xi^{+}$yield in Au+Au collisions at $160 A \mathrm{GeV}$ as calculated with the hadronic HSD model and the pHSD model including a QGP phase, as function of impact parameter [17]

can be observed. While the calculations from Lattice QCD are performed for infinite matter at equilibrium, the real system is finite in both lifetime and size. There are thus no quantitative predictions for the critical fluctuations to be expected.

Collider experiments are best suited for the systematic search of non-statistical fluctuations because of their uniform azimuthal acceptance, which does not vary significantly with collision energy, and their larger range in $\sqrt{s}$. Thus, the second phase of the BES, planned for 2018 with increased luminosity and, consequently, significantly improved event statistics, is most promising. Similarly, fluctuation data can be expected from the MPD experiment at NICA, covering the beam energy range where the luminosity of the RHIC collider does not allow precision measurements.

\subsection{Vector mesons}

An observable consequence of the restoration of chiral symmetry would be a modification of hadron properties inside nuclei, or in hot and dense matter. Short-lived vector mesons, in particular the $\rho$, decay inside the fireball and thus preserve their state in the decay kinematics. Although their branching ratio to lepton pairs is small, this decay channel is the experimentally interesting one, since the leptons, not strongly interacting, leave the reaction zone undisturbed and thus carry the information on the vector meson properties. The "melting of the $\rho$ " was indeed observed at top SPS energy [18]. However, results from the HADES experiment show that the excess of low-mass lepton pairs over the expectations from a "conventional" hadronic cocktail is even larger at very low energies [19], as shown in Fig. 6. This result has raised doubts as to the relation of the observed $\rho$ melting with chiral restoration. For a better understanding, systematic measurements in the energy region between SIS-18 and top SPS are indispensable.

Of similar interest is the di-lepton mass region between the $\phi$ and the $J / \psi$ meson, since here, no hadronic sources contribute directly. Thus, the lepton pairs in this mass region are results of direct radiation from the hot collision zone. Their transverse mass spectrum gives access to the apparent temperature of the medium, reflecting both the true temperature and the blue shift due to collective motion. The pioneering studies of the NA60 collaboration show a constant apparent temperature for pairs above $1 \mathrm{GeV}$ invariant mass, consistent with an early source with moderate transverse flow (see Fig. 7). This exciting result clearly calls for extending such measurements to lower energies. 
A renewal of the NA60 experiment as discussed in Ref. [7] is therefore quite appealing a project. Experimental data on lepton pairs can also be expected from the MPD experiment in the di-electron channel, from the continuation of the HADES experiment at the FAIR facility (up to $4 A \mathrm{GeV}$ for $\mathrm{Au}+\mathrm{Au}$ and $8 A \mathrm{GeV}$ for lighter systems [21]), and, finally, from the CBM experiment in both the di-muon and di-electron channel over the entire FAIR energy range.

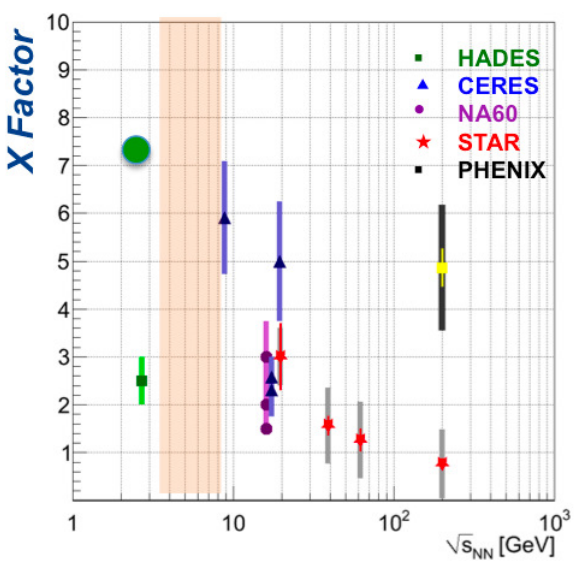

Figure 6: Excess of low-mass di-lepton yield over the hadronic cocktail as function of collision energy (compilation by T. Galatyuk)

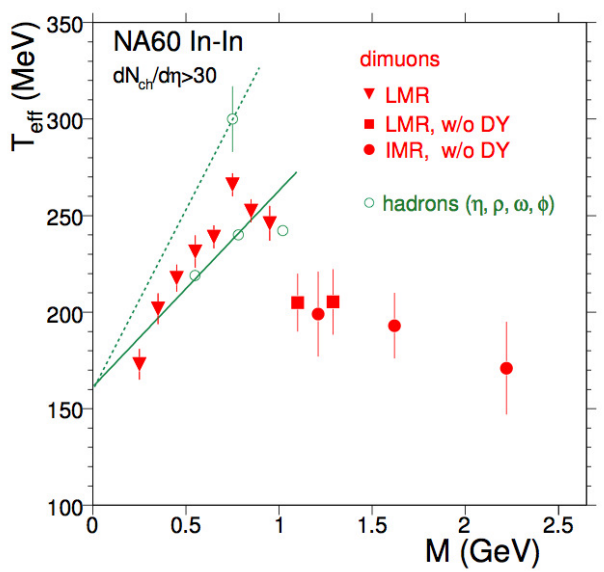

Figure 7: Apparent temperature of muon pairs as function of their invariant mass, measured in $\mathrm{In}+\mathrm{In}$ collisions at $158 \mathrm{~A} \mathrm{GeV} \mathrm{[20]}$

\subsection{Charm}

Hadrons containing charm quarks are very promising diagnostic probes of hot and dense nuclear matter. Because of their large mass, charm quarks cannot be thermally produced in nucleus-nucleus reactions, but are created in the initial stage by first-chance collisions of the participating nucleons. They then propagate through the dense medium. Hadrons containing charm quarks are thus very promising diagnostic probes of hot and dense nuclear matter. This argument holds independently of the collision energy, provided it is large enough to produce charm at all.

If the produced medium is partonic, the formation of charmonium states will be suppressed by Debye screening, and the charm quarks will finally coalesce with light quarks to hadrons with open charm. This "anomalous" suppression of the $J / \psi$ yield relative to muon pairs from Drell-Yan processes was observed by the NA50 collaboration in central $\mathrm{Pb}+\mathrm{Pb}$ collisions at $158 \mathrm{~A} \mathrm{GeV}$ [22], providing one of the strongest arguments for a QGP formation at top SPS energy [14]. It is therefore natural to look for the disappearance of this signal at lower collision energies, where the energy density is not sufficient to create a QGP. The interpretation of data from this energy domain would be more straightforward than at RHIC and LHC energies, since charm regeneration in an equilibrated medium is negligible here. However, for the interpretation of the charmonium data, a knowledge on the total charm cross section is required, which necessitates also the measurement of open charm hadrons. Experimentally, the charm cross section below $\sqrt{s}=20 \mathrm{GeV}$ is unknown even in elementary reactions, and no data on neither hidden nor open charm exist from heavy-ion reactions below top SPS energy. 
Model calculations confirm that charmed hadrons are a promising indicator of their production mechanism and thus of the medium they have been created in. Figure 9 shows the ratio of the $J / \psi$ and $D+\bar{D}$ yields in Au+Au collisions as function of collision energy, both for the hadronic transport model HSD [23] and for the statistical model [24], which assumes thermalisation of charm quarks and thus implicitly the presence of a deconfined medium. The yield ratios of the two scenarios differ by factors, which should be experimentally well assessable.

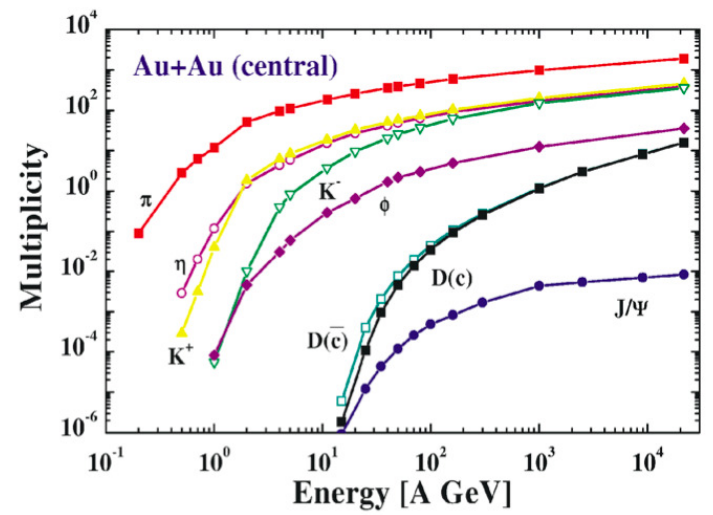

Figure 8: Predictions of the HSD model for the production of charmed hadrons in central $\mathrm{Au}+\mathrm{Au}$ collisions as a function of beam energy [23]

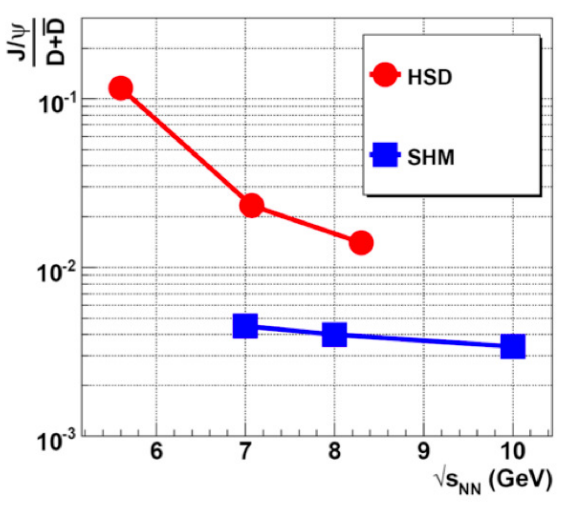

Figure 9: Ratio of hidden to open charm as calculated with the HSD model [23] and the statistical model [24] as function of collision energy

The experimental challenge lies in the extremely low abundances of both open and hidden charm at lower collision energies. In addition, micro-vertexing capabilities are required to identify $\mathrm{D}$ mesons by their displaced decay vertex. While NA61 with an additional vertex detector has a chance to measure open charm down to $80 \mathrm{~A} \mathrm{GeV} \mathrm{[4],} \mathrm{a} \mathrm{possible} \mathrm{NA60+} \mathrm{experiment} \mathrm{could} \mathrm{measure} \mathrm{charmonium} \mathrm{down}$ to $60 \mathrm{~A} \mathrm{GeV} \mathrm{[7].} \mathrm{A} \mathrm{systematic} \mathrm{investigation} \mathrm{of} \mathrm{charm} \mathrm{production} \mathrm{at} \mathrm{even} \mathrm{lower} \mathrm{energies} \mathrm{will} \mathrm{only}$ be possible with the CBM experiment [6], being designed to cope with the extreme interaction rates necessary to access such rare observables.

\section{Summary}

Substantial experimental efforts worldwide are ongoing to investigate strongly interacting matter at high densities by the study of nuclear collisions at moderate and low energies. These efforts are parallel and complementary to the activities at top RHIC energy and at the LHC, which study deconfined QCD matter at the highest available energy densities, but vanishing matter density. Their goal is to detect the landmarks of the QCD phase diagram at high density: the first-oder deconfinement phase transition, the restoration of chiral symmetry, and the QCD critical point. Although guidelines from theory are less well established in this regime because of the inapplicability of ab-initio QCD calculations, many observables can be identified which are promising diagnostics for the state of the matter created in such nuclear collisions. Some of the experimental programmes are already ongoing at existing accelerators; others rely on dedicated next-generation experiments to be operated at upcoming facilities from about 2020 on, coincidentally parallel to run 3 of the LHC. The future of heavy-ion physics in the next decade thus looks bright and promising. 


\section{References}

[1] For an overview, see C. DeTar and U. M. Heller, Eur. Phys. J. A 41, 405 (2009)

[2] I. Arsene et al. (BRAHMS collab.), Nucl. Phys. A 7571 (2005);

B. Back et al. (PHOBOS collab.), Nucl. Phys. A 75728 (2005);

J. Adams et al. (STAR collab.), Nucl. Phys. A 757102 (2005);

K. Adcox et al. (PHENIX collab.), Nucl. Phys. A 757184 (2005)

[3] D. McDonald for the STAR collaboration, this volume

[4] M. Gazdzicki for the NA61 collaboration, this volume

[5] A. Sorin for the NICA/MPD collaboration, this volume

[6] J. Heuser for the CBM collaboration, this volume

[7] G. Usai, this volume

[8] H. Sako et al., Nucl. Phys. A 931, 1158 (2014)

[9] K. Fukushima and T. Hatsuda, Rept. Prog. Phys. 74, 014001 (2011)

[10] A. Andronic et al., Nucl. Phys. A 837, 65 (2010)

[11] A. Andronic et al., Phys. Lett. B B 673, (2009)

[12] B. Friman et al. (Eds.), The CBM Physics Book, Springer Lect. Notes in Physics 814, 641 (2011)

[13] J. Randrup and J. Cleymans, Phys. Rev. C 74, 047901 (2006)

[14] CERN press release 10 February 2000, http://press.web.cern.ch/press-releases/2000/02/ new-state-matter-created-cern;

U. Heinz, arxiv:nucl-th/0002042

[15] G. Agakishiev et al. (HADES collaboration), Eur. Phys. J. A 47, 21 (2011)

[16] C. Alt et al. (NA49 collaboration), Phys. Rev. C 77, 024903 (2008)

[17] W. Cassing, priv. comm.

[18] S. Damjanovic et al. (NA60 collaboration), Nucl. Phys. A A 783, 327 (2007)

[19] T. Galatyuk for the HADES collaboration, Nucl. Phys. A A 931, 41 (2014)

[20] H. J. Specht for the NA60 collaboration, AIP Conf. Proc. 13221 (2010)

[21] P. Salabura for the HADES collaboration, this volume

[22] B. Alessandro et al. (NA50 collaboration), Eur. Phys. J. C 39, 335 (2005)

[23] W. Cassing et al., Nucl. Phys. A 6917513 (2001)

[24] A. Andronic et al., Phys. Lett. B 659, 149 (2008) 